\title{
Another abstraction of the Erdős-Szekeres Happy End Theorem
}

\author{
Noga Alon* $\quad$ Ehsan Chiniforooshan ${ }^{\dagger} \quad$ Vašek Chvátal ${ }^{\ddagger}$ \\ François Genest §
}

Submitted: Jul 13, 2009; Accepted: Jan 26, 2010; Published: Feb 8, 2010

Mathematics Subject Classification: 05D10

\begin{abstract}
The Happy End Theorem of Erdős and Szekeres asserts that for every integer $n$ greater than two there is an integer $N$ such that every set of $N$ points in general position in the plane includes the $n$ vertices of a convex $n$-gon. We generalize this theorem in the framework of certain simple structures, which we call "happy end spaces".
\end{abstract}

In the winter of 1932/33, Esther Klein observed that

from any set of five points in the plane of which no three lie on the same line it is always possible to select four points that are vertices of a convex polygon.

When she shared this news with a circle of her friends in Budapest, the following prospect of generalizing it emerged:

Can we find for each integer $n$ greater than two an integer $N(n)$ such that from any set of $N(n)$ points in the plane of which no three lie on the same line it is always possible to select $n$ points that are vertices of a convex polygon?

Endre Makai proved that $N(5)=9$ works here. A few weeks later, George Szekeres proved the existence of $N(n)$ for all $n$. His argument produced very large upper bounds for $N(n)$ : for instance, it gave $N(5) \leqslant 2^{10000}$. Soon afterwards, Paul Erdős came up with a different proof, which led to much smaller values of $N(n)$ :

\footnotetext{
* Sackler School of Mathematics and Blavatnik School of Computer Sciences, Tel Aviv University, Tel Aviv, Israel

${ }^{\dagger}$ Department of Computer Science and Software Engineering, Concordia University, Montréal, Québec, Canada

${ }_{\ddagger}^{\ddagger}$ Department of Computer Science and Software Engineering, Concordia University, Montréal, Québec, Canada

$\S 5264$ av. Henri-Julien app. 3, Montréal, Québec, Canada
} 
(*) From any set of $\left(\begin{array}{c}2 n-4 \\ n-2\end{array}\right)+1$ points in the plane of which no three lie on the same line it is always possible to select $n$ points that are vertices of a convex polygon.

In December 1934, Erdős and Szekeres submitted for publication a manuscript containing both proofs; the paper [6] appeared in 1935. Esther Klein and George Szekeres got married on June 13, 1937 and Paul Erdős began referring to (*) as The Happy End Theorem.

Abstractions of this theorem have been studied by Korte and Lovász [7] and by Morris and Soltan [8]. These deal with abstract convexity spaces satisfying the anti-exchange property and the simplex partition property, and having a finite Caratheodory number $c \geqslant 3$. See [8], [7] for the precise definitions and more details. A somewhat less abstract version is considered in [10], based on the order type of a configuration of points in the plane. This enables the authors to show, using an exhaustive computer search, that any configuration of 17 points in general position in the plane contains a convex 6-gon. Here we propose another abstraction: A happy-end space is a set $S$ along with a function

$$
f: S \times S \times S \rightarrow\{+,-\}
$$

such that

$$
f(x, y, z)=f(y, x, z) \text { for all } x, y, z \text {; }
$$

in this space, a subset $C$ of $S$ is called convex if, and only if, for every subset $B$ of $C$ such that $|B|>2$ and for every point $x$ of $B$, there is another $y$ in $B$ such that $f(x, y, z)$ is constant on $B-\{x, y\}$. (In this definition, the only values of $f(x, y, z)$ that matter are those where $x, y, z$ are all distinct.) Every set $S$ of points in the plane such that no three lie on the same line and no two have the same first coordinate defines a happy-end space by

$$
f(x, y, z)= \begin{cases}+1 & \text { if point } z \text { lies above the line } \overline{x y} \\ -1 & \text { if point } z \text { lies below the line } \overline{x y}\end{cases}
$$

in this space, a set is convex if and only if it consists of vertices of a convex polygon.

In this abstract setting, the Happy End Theorem generalizes as follows:

Theorem 1. For every positive integer $n$ there is a positive integer $N$ such that every happy-end space on $N$ points contains a convex set of $n$ points.

Proof. Following Erdős and Rado [4, 5], we let $a \rightarrow(b)_{r}^{k}$ denote the statement that whenever the $k$-point subsets of an $a$-point set are coloured by $r$ colours, there is a $b$-point set whose $k$-point subsets are all of the same colour.

Frank Ramsey [9] proved that

for every choice of positive integers $b, k, r$, there is an integer $a$ such that $a \rightarrow(b)_{r}^{k}$. 
We claim that if $N$ satisfies $N \rightarrow(n)_{8}^{3}$ then it also satisfies the conclusion of Theorem 1 .

To justify this claim, consider an arbitrary happy-end space on $N$ points, impose a linear order $\prec$ on its underlying set $S$ and, for each set $T$ of three points in $S$, write

$$
g(T)=(f(v, w, u), f(u, w, v), f(u, v, w)) \text {, where } T=\{u, v, w\} \text { and } u \prec v \prec w .
$$

Since $N \rightarrow(n)_{8}^{3}$, there are a set $C$ of $n$ points in $S$ and a vector $\left(s_{1}, s_{2}, s_{3}\right)$ in $\{+1,-1\}^{3}$ such that $g(T)=\left(s_{1}, s_{2}, s_{3}\right)$ for every three-point subset $T$ of $C$; this means that

$$
f(x, y, z)= \begin{cases}s_{1} & \text { if } z \prec x \prec y \text { or } z \prec y \prec x, \\ s_{2} & \text { if } x \prec z \prec y \text { or } y \prec z \prec x, \\ s_{3} & \text { if } x \prec y \prec z \text { or } y \prec x \prec z .\end{cases}
$$

We are going to show that $C$ is convex. For this purpose, consider an arbitrary subset $B$ of $C$ such that $|B|>2$ and enumerate its points as $u_{1}, u_{2}, \ldots, u_{k}$ in such a way that $u_{1} \prec u_{2} \prec \ldots \prec u_{k}$. Given any $u_{i}$ in $B$, we will find another $u_{j}$ in $B$ such that $f\left(u_{i}, u_{j}, z\right)$ is constant on $B-\left\{u_{i}, u_{j}\right\}$.

CASE 1: $s_{1}=s_{2}$. In this case, we may set $j=k$ unless $i=k$, in which case any $j$ smaller than $k$ will do. It is not difficult to check that with this choice of $j$, $f\left(u_{i}, u_{j}, u_{r}\right)=s_{1}\left(=s_{2}\right)$ for all $u_{r} \in B-\left\{u_{i}, u_{j}\right\}$.

CASE 2: $s_{2}=s_{3}$. In this case, we may set $j=1$ unless $i=1$, in which case any $j$ greater than 1 will do. With this choice, $f\left(u_{i}, u_{j}, u_{r}\right)=s_{2}\left(=s_{3}\right)$ for all $u_{r} \in B-\left\{u_{i}, u_{j}\right\}$.

CAsE 3: $s_{1} \neq s_{2}$ and $s_{2} \neq s_{3}$. In this case, $s_{1}=s_{3}$; we may set $j=i+1$ unless $i=k$ and we may set $j=i-1$ unless $i=1$. This ensures that $f\left(u_{i}, u_{j}, u_{r}\right)=s_{1}\left(=s_{3}\right)$ for all $u_{r} \in B-\left\{u_{i}, u_{j}\right\}$.

We have found the proof of Theorem 1 during the 7 th International Colloquium on Graph Theory in Hyères in September 2005. Another proof of the theorem was found, independently, by Pierre Duchet during the same conference [3]. His proof also uses Ramsey's theorem, but it gives a far larger upper bound for $N$.

Let $N_{0}(n)$ denote the smallest integer $N$ such that from any set of $N$ points in the plane of which no three lie on the same line it is always possible to select $n$ points that are vertices of a convex polygon. As shown in [6], $N_{0}(n) \geqslant 2^{n-2}+1$ for every $n \geqslant 3$, and the authors of [6] conjectured that this is tight for all $n$.

The conjecture is open for all $n>6$. Regarding upper bounds, the Happy End Theorem asserts that

$$
N_{0}(n) \leqslant\left(\begin{array}{c}
2 n-4 \\
n-2
\end{array}\right)+1
$$

in 1998, Chung and Graham [2] improved this upper bound to

$$
N_{0}(n) \leqslant\left(\begin{array}{c}
2 n-4 \\
n-2
\end{array}\right)
$$

subsequently, Tóth and Valtr [11] reduced it to roughly its half,

$$
N_{0}(n) \leqslant\left(\begin{array}{c}
2 n-5 \\
n-2
\end{array}\right)+1
$$


Chung and Graham [1] have offered $\$ 100$ for the first proof that $N_{0}(n)=O\left(c^{n}\right)$ for some constant $c$ smaller than 4 .

Our second theorem shows that even the original Erdős-Szekeres upper bound on $N_{0}(n)$ grows much too slowly with $n$ to be applicable in the more general setting of happy-end spaces.

Theorem 2. For every positive integer $n$ there is a happy-end space on $2^{\Omega\left(n^{2}\right)}$ points that contains no convex set of $n$ points.

It is worth noting that the upper bound that follows from the proof of Theorem 1 and the known bounds for hypergraph Ramsey numbers is doubly exponential in $n$, hence there is still a substantial gap between the upper and lower bounds proved here.

Proof. Given a positive integer $n$, set

$$
N=\left\lfloor\left(\frac{\sqrt{e}}{2 \sqrt{n}} \cdot 2^{n / 6}\right)^{n}\right\rfloor
$$

We will prove that in a happy-end space taken uniformly at random from all possible happy-end spaces on $N$ points, the expected number of convex sets of size $n$ is less than 1. For this purpose, we will bound from above the probability $p_{n}$ that a prescribed set of size $n$ in this happy-end space is convex.

If a set $C$ of size $n$ is convex, then, for each of its points $x_{1}$,

(i) the $n-1$ points of $C-\left\{x_{1}\right\}$ can be enumerated as $x_{2}, x_{3}, \ldots, x_{n}$ in such a way that, for each $j=2,3, \ldots, n-2$, the $n-j$ values of $f\left(x_{1}, x_{j}, x_{k}\right)$ with $k=j+1, j+2, \ldots n$ are identical,

(ii) $C-\left\{x_{1}\right\}$ is convex.

The probability of (i) is at most $(n-1) ! \prod_{j=2}^{n-2}\left(\frac{1}{2}\right)^{n-j-1}$; since events (i) and (ii) are independent, we conclude that

$$
p_{n} \leqslant\left((n-1) ! \prod_{j=2}^{n-2}\left(\frac{1}{2}\right)^{n-j-1}\right) p_{n-1}=\left((n-1) !\left(\frac{1}{2}\right)^{(n-2)(n-3) / 2}\right) p_{n-1} .
$$

Since $p_{3}=1$ and $m !<(m / e)^{m}$ and $(n-1)(n-2)(n-3)>n^{3}-6 n^{2}$, it follows that

$$
p_{n} \leqslant\left(\prod_{m=3}^{n-1} m !\right)\left(\frac{1}{2}\right)^{(n-1)(n-2)(n-3) / 6}<\left(\frac{2 \sqrt{n}}{\sqrt{e}}\left(\frac{1}{2}\right)^{n / 6}\right)^{n^{2}} \leqslant \frac{1}{N^{n}} .
$$

Finally, we note that the expected number of convex sets of size $n$ equals $\left(\begin{array}{l}N \\ n\end{array}\right) p_{n}$ and that $\left(\begin{array}{l}N \\ n\end{array}\right)=o\left(N^{n}\right)$. 
Let $N_{1}(n)$ denote the smallest integer $N$ that works in Theorem 1: in this notation, Theorem 2 states that $N_{1}(n)=2^{\Omega\left(n^{2}\right)}$. By definition, $N_{0}(n) \leqslant N_{1}(n)$ for all $n$; trivially, $N_{0}(3)=N_{1}(3)=3$; Esther Klein's observation was that $N_{0}(4)=5$. In closing, we note that

$$
8 \leqslant N_{1}(4) \leqslant 11
$$

To see that $N_{1}(4) \leqslant 11$, consider an arbitrary happy-end space $(S, f)$ such that $|S|=$ 11. Choose two distinct points $u, v$ of $S$. By the pigeon-hole principle, there is a 5-point subset $R$ of $S-\{u, v\}$ such that $f(u, v, t)$ is constant as $t$ ranges through $R$. Choose a point $w$ in $S-(\{u, v\} \cup R)$. By the pigeon-hole principle again, there are first a 3-point subset $Q$ of $R$ such that $f(u, w, t)$ is constant as $t$ ranges through $Q$, then distinct points $x, y$ in $Q$ such that $f(v, w, x)=f(v, w, y)$, and finally distinct points $z, z^{\prime}$ in $\{u, v, w\}$ such that $f(x, y, z)=f\left(x, y, z^{\prime}\right)$. It is now not too difficult to check that the set $\left\{x, y, z, z^{\prime}\right\}$ is convex. Indeed, by the construction above $f(u, v, x)=f(u, v, y), f(u, w, x)=f(u, w, y)$ and $f(v, w, x)=f(v, w, y)$. By symmetry, we may assume without loss of generality that $z, z^{\prime}$ above are $u, v$, and thus $f(x, y, u)=f(x, y, v)$. To show that the set $S=\{x, y, u, v\}$ is convex we have to check that for every element $a \in S$ there is another element $b \in S$, so that $f(a, b, c)$ is constant on the two elements $c \in S-\{a, b\}$. Indeed, for $a=x$ take $b=y$ and vice versa, and for $a=u$, take $b=v$ and vice versa, as needed.

To see that $N_{1}(4) \geqslant 8$, consider the happy-end space $(\{0,1, \ldots, 6\}, f)$ with the values of $f(0, y, z)$ given by the table

$$
\begin{array}{lccccccc} 
& z=1 & z=2 & z=3 & z=4 & z=5 & z=6 \\
f(0,1, z)= & & + & - & - & + & + \\
f(0,2, z)= & - & & + & + & + & - \\
f(0,3, z)= & - & + & & + & - & + \\
f(0,4, z)= & + & - & + & & - & + \\
f(0,5, z)= & + & + & + & - & & - \\
f(0,6, z)= & + & - & - & + & + &
\end{array}
$$

and the remaining values of $f(x, y, z)$ determined by the circular symmetry $f(x, y, z)=$ $f(x+1, y+1, z+1)$ with the increment modulo 7. ( In particular, $f(4,0, z)=f(0,3, z+3)$, $f(5,0, z)=f(0,2, z+2), f(6,0, z)=f(0,1, z+1)$, and so the last three rows of the table are redundant. We include them for the ease of checking the argument that follows.) A four-point set $R$ is not convex if and only if it has an element $x$ such that, for each way of enumerating the remaing three elements of $R$ as $y, z, z^{\prime}$, we have $f(x, y, z) \neq f\left(x, y, z^{\prime}\right)$. Let us call such an $x$ an interior point of $R$. In our happy-end space, point 0 is the interior point of the five sets

$$
\{0,1,2,3\},\{0,1,2,4\},\{0,1,4,5\},\{0,2,4,6\},\{0,3,5,6\}
$$

the circular symmetry guarantees that each of $1,2, \ldots, 6$ is the interior point of an additional five four-points sets, and so each of the 35 four-point subsets of $\{0,1, \ldots, 6\}$ has an interior point. 


\section{Acknowledgment}

The work of the first author was supported, in part, by an ISF grant, by an ERC advanced grant and by the Hermann Minkowski Minerva Center for Geometry at Tel Aviv University. The work of the last three authors was carried out in ConCoCO (Concordia Computational Combinatorial Optimization Laboratory) and undertaken, in part, thanks to funding from the Canada Research Chairs Program and from the Natural Sciences and Engineering Research Council of Canada.

\section{References}

[1] F.R.L. Chung and R.L. Graham, Erdös on Graphs. His Legacy of Unsolved Problems, A.K. Peters, Ltd., Wellesley, MA, 1998.

[2] F.R.L. Chung and R.L. Graham, Forced convex $n$-gons in the plane, Discrete Comput. Geom. 19 (1998), 367-371.

[3] P. Duchet, Private communication.

[4] P. Erdős and R. Rado, A problem on ordered sets, J. London Math. Soc. 28 (1953), 426-438.

[5] P. Erdős and R. Rado, A partition calculus in set theory, Bull. Amer. Math. Soc. 62 (1956), 427-489.

[6] P. Erdős and G. Szekeres, A combinatorial problem in geometry, Compositio Math. 2 (1935), 463-470.

[7] B. Korte and L. Lovász, Shelling structures, convexity and a happy end. In: B. Bollobás, ed., Graph Theory and Combinatorics (Cambridge, 1983), 219-232, Academic Press, London, 1984.

[8] W. Morris and V. Soltan, The Erdös-Szekeres problem on points in convex position - a survey, Bull. Amer. Math. Soc. (N.S.) 37 (2000), 437-458.

[9] F.P. Ramsey, On a problem of formal logic, Proc. London Math. Soc. 30 (1930), $361-376$.

[10] G. Szekeres and L. Peters, Computer solution to the 17-point Erdős-Szekeres problem, The ANZIAM Journal 48 (2006), 151-164.

[11] G. Tóth and P. Valtr, The Erdös-Szekeres theorem: upper bounds and related results. In: J.E. Goodman, J.Pach and E. Welzl, eds., Combinatorial and Computational Geometry Vol. 19, MSRI Publications (2005), pp. 557-568. 